\title{
REAL ZERO POLYNOMIALS AND PÓLYA-SCHUR TYPE THEOREMS
}

\author{
By
}

Alexandru Aleman, Dmitry Beliaev and HaAkan Hedenmalm

\section{Introduction}

Real zero preserving operators. Let $\mathcal{P}(\mathbb{C})$ denote the space of all polynomials with complex coefficients, regarded as functions on the complex plane. The differentiation operator $D=d / d z$ acts on $\mathcal{P}(\mathbb{C})$; the action on the monomials is given by $D z^{n}=n z^{n-1}$ for $n=1,2,3, \ldots$. We also have the operator $D_{*}$ of multiplicative differentiation, related to $D$ via $D_{*}=z D$; the action on the monomials is given by $D_{*} z^{n}=n z^{n}$ for $n=0,1,2, \ldots$. Whereas $D$ commutes with all translations, $D_{*}$ commutes with all dilations of the complex plane $\mathbb{C}$. A third differentiation operator $D_{\sharp}=z^{2} D$ is of interest; its action on the monomials is given by $D_{\sharp} z^{n}=n z^{n+1}$ for $n=0,1,2, \ldots$ We get it by first inverting the plane $(z \mapsto 1 / z)$, then applying minus differentiation $-D$, and by finally inverting back again. This means that studying $D_{\sharp}$ on the polynomials is equivalent to studying the ordinary differentiation operator $D$ on the space of all rational functions that are regular at all points of the extended plane with the exception of the origin.

The Gauss-Lucas theorem states that if a polynomial $p(z)$ has its zeros contained in some given convex set $K$, then its derivative $D p(z)=p^{\prime}(z)$ has all its zeros in $K$ as well (unless $p(z)$ is constant). In particular, if all the zeros are real, then so are the zeros of the derivative. Naturally, the same statement can be made for the multiplicative derivative $D_{*}$ as well, and for $D_{\sharp}$, too. In this context, we should mention the classical theorem of Laguerre [2, p. 23], which extends the GaussLucas theorem for the real zeros case to the more general setting of entire functions of genus 0 or 1 . To simplify the later discussion, we introduce the notation $\mathcal{P}(\mathbb{C} ; \mathbb{R})$ for the collection of all polynomials with only real zeros, including all constants. This means that the zero polynomial is in $\mathcal{P}(\mathbb{C} ; \mathbb{R})$, although, strictly speaking, it has plenty of non-real zeros. Clearly, $\mathcal{P}(\mathbb{C} ; \mathbb{R})$ constitutes a multiplicative semigroup. Let $T: \mathcal{P}(\mathbb{C}) \rightarrow \mathcal{P}(\mathbb{C})$ be a linear operator. Let us say that $T$ is real zero 
preserving if $T(\mathcal{P}(\mathbb{C} ; \mathbb{R})) \subset \mathcal{P}(\mathbb{C} ; \mathbb{R})$. It would be of interest to have a complete characterization of the real zero preserving operators. From the above remarks, we know that $D, D_{*}$, and $D_{\sharp}$ are real zero preserving. To get some headway into the general problem, it is helpful to have some additional information regarding the given operator $T$.

The Pólya-Schur theorem for multiplicative differentiation. A natural condition is that $T$ should commute with a given "good" operator. This was worked out by Pólya and Schur in 1914 [7], [6], [5, pp. 100-124], [8, pp. 56-69, pp. 88112 ] in the case that $T$ commutes with $D_{*}$; it is a simple exercise to check that such a $T$ has the following action on the monomials:

$$
T\left[z^{n}\right]=\tau_{n} z^{n}, \quad n=0,1,2, \ldots,
$$

where the "eigenvalues" $\tau_{n}$ are complex constants. The description is in terms of the function

$$
\Phi_{T}(z)=T\left[e^{z}\right]=\sum_{n=0}^{+\infty} \frac{\tau_{n}}{n !} z^{n},
$$

which is - so far - only a formal power series. To formulate the Pólya-Schur result, we need to understand which functions occur as limits of polynomials with only real zeros. An entire function $f(z)$ is said to belong to the Laguerre-Pólya class $\operatorname{LP}(\mathbb{C} ; \mathbb{R})$ if it can be written in the form

$$
f(z)=C e^{\alpha z-\beta z^{2}} z^{N} \prod_{k}\left(1-\frac{z}{x_{k}}\right) e^{z / x_{k}}, \quad z \in \mathbb{C},
$$

where $\alpha, \beta$ are real with $0 \leq \beta<+\infty, C$ is a nonzero complex number, $N$ is a nonnegative integer, and $\left\{x_{k}\right\}_{k}$ is a finite or infinite sequence of nonzero real numbers with $\sum_{k} x_{k}^{-2}<+\infty$. The number $\beta$ above will be referred to as the order two characteristic of the function $f$. It is a theorem of Pólya [4], [5, pp. 54-70] that (a) each function $f(z)$ in $\operatorname{LP}(\mathbb{C} ; \mathbb{R})$ is the limit of polynomials with only real zeros in the topology of uniform convergence on compact subsets, and that (b) if a sequence of polynomials with only real zeros converges uniformly on any neighborhood of the origin, then either the limit vanishes identically or the limit is an entire function in $\operatorname{LP}(\mathbb{C} ; \mathbb{R})$. There is also the related Laguerre-Pólya class $\operatorname{LP}\left(\mathbb{C} ; \mathbb{R}_{+}\right)$, which consists of all functions $f$ of the form

$$
f(z)=C e^{\alpha z} z^{N} \prod_{k}\left(1-\frac{z}{x_{k}}\right), \quad z \in \mathbb{C},
$$

where $-\infty<\alpha \leq 0, C$ is a nonzero complex number, $N$ is a nonnegative integer, and all $x_{k}$ 's are positive with $\sum_{k} x_{k}^{-1}<+\infty$. It can be characterized as the 
nontrivial limits of polynomials with positive real zeros. We also need the class $\mathrm{LP}\left(\mathbb{C}_{;} \mathbb{R}_{-}\right)$of entire functions $f(z)$ such that $f(-z)$ belongs to $\operatorname{LP}\left(\mathbb{C}_{;} \mathbb{R}_{+}\right)$, which then is the limit space for polynomials with negative real zeros.

Theorem 1.1 (Pólya-Schur). Let $T: \mathcal{P}(\mathbb{C}) \rightarrow \mathcal{P}(\mathbb{C})$ be a linear operator which commutes with $D_{*}$. Then $T$ preserves real zeros if and only if the associated function $\Phi_{T}(z)$ defined by (1.2) belongs to the set $\operatorname{LP}\left(\mathbb{C}_{;}, \mathbb{R}_{+}\right) \cup \operatorname{LP}\left(\mathbb{C}_{;} \mathbb{R}_{-}\right) \cup\{0\}$. Likewise, $T$ maps positive real zeros (or negative real zeros) to real zeros if and only if $\Phi_{T}(z)$ belongs to $\mathrm{LP}(\mathbb{C} ; \mathbb{R}) \cup\{0\}$.

In the theorem, to map positive real zeros to real zeros means that $T\left(\mathcal{P}\left(\mathbb{C}_{;} \mathbb{R}_{+}\right)\right) \subset$ $\mathcal{P}(\mathbb{C} ; \mathbb{R})$, where $\mathcal{P}\left(\mathbb{C} ; \mathbb{R}_{+}\right)$is the multiplicative semigroup of all polynomials with only positive real zeros (including the zero polynomial); to map negative real zeros to real zeros is defined analogously. It is not hard to show that in the setting of the above theorem, with $T$ a real zero preserving operator, $T$ extends in a continuous fashion to an operator that leaves the multiplicative semigroup $\operatorname{LP}(\mathbb{C} ; \mathbb{R}) \cup\{0\}$ invariant. The criterion that $\Phi_{T} \in \operatorname{LP}\left(\mathbb{C}_{;} \mathbb{R}_{+}\right) \cup \operatorname{LP}\left(\mathbb{C}_{\mathbb{R}} \mathbb{R}_{-}\right) \cup\{0\}$ is equivalent to the following three requirements:

$T\left[e^{z}\right] \in \operatorname{LP}(\mathbb{C} ; \mathbb{R}) \cup\{0\}, \quad T\left[e^{-z^{2}}\right] \in \operatorname{LP}(\mathbb{C} ; \mathbb{R}) \cup\{0\}, \quad T\left[z e^{-z^{2}}\right] \in \operatorname{LP}(\mathbb{C} ; \mathbb{R}) \cup\{0\}$.

In other words, by the theorem, the process of checking that $T(\mathcal{P}(\mathbb{C} ; \mathbb{R})) \subset \mathcal{P}(\mathbb{C} ; \mathbb{R})$ reduces to verifying that $T[f] \in \operatorname{LP}(\mathbb{C} ; \mathbb{R}) \cup\{0\}$ for three functions $f$ in $\operatorname{LP}(\mathbb{C} ; \mathbb{R})$ : $f(z)=e^{z}, f(z)=e^{-z^{2}}$, and $f(z)=z e^{-z^{2}}$. We can think of these functions as "test points" for the problem. We should mention here that after multiplication by an appropriate complex constant, we may split the condition $\Phi_{T} \in \operatorname{LP}\left(\mathbb{C}_{;} \mathbb{R}_{-}\right)$ appearing in Theorem 1.1 in two conditions, namely, $\Phi_{T} \in \operatorname{LP}(\mathbb{C} ; \mathbb{R})$ and that the coefficients $\left\{\tau_{j}\right\}_{j}$ defined by (1.2) be nonnegative. A similar criterion characterizes $\Phi_{T} \in \operatorname{LP}\left(\mathbb{C}_{;} \mathbb{R}_{+}\right)$in terms of sign alternating coefficients $\left\{\tau_{j}\right\}_{j}$.

The Pólya-Benz theorem for additive differentiation. Here, we consider operators $T: \mathcal{P}(\mathbb{C}) \rightarrow \mathcal{P}(\mathbb{C})$ which commute with ordinary (additive) differ. entiation $D$. An operator $T$ which commutes with $D$ has a power series expansion

$$
T=\sum_{n=0}^{+\infty} \widehat{T}(n) D^{n},
$$

where the coefficients $\widehat{T}(n)$ are complex numbers; we observe that if we apply the series to a polynomial,

$$
T p(z)=\sum_{n=0}^{+\infty} \widehat{T}(n) D^{n} p(z)
$$


only finitely many terms in the series are nonzero, so the lack of growth control of the coefficients in the power series expansion causes no problems. The symbol of the operator $T$ is the (formal) power series

$$
F_{T}(z)=\sum_{n=0}^{+\infty} \widehat{T}(n) z^{n} .
$$

We formulate the Pólya-Benz theorem [1].

Theorem 1.2 (Pólya-Benz). Let $T: \mathcal{P}(\mathbb{C}) \rightarrow \mathcal{P}(\mathbb{C})$ be a linear operator which commutes with $D$, the differentiation operator.' Then $T$ preserves real zeros if and only if $F_{T}(z)$ is an entire function which is either the zero function or belongs to the Laguerre-Pólya class $\mathrm{LP}(\mathbb{C} ; \mathbb{R})$.

This theorem is more elementary than the Pólya-Schur theorem, although it apparently was found later. However, at least part of the theorem seems to trace its way back to Laguerre. The point we wish to make here is that in contrast to the Pólya-Schur theorem, there is no actual function that we should check $T$ on to get the criterion of the theorem, but rather a sequence of functions, the monomials $p(z)=z^{N}$, for $N=0,1,2,3, \ldots$, and we should let $N$ approach $+\infty$. Nevertheless, it makes sense to say that the criterion checks the action of $T$ at a single improper "test point", which we think of as the limit (in some sense) of the monomials $z^{N}$ as $N \rightarrow+\infty$.

In the context of Theorem 1.2, it is possible to extend the action of the real zero preserving operator $T$ continuously in such a way that $T[f]$ remains a well-defined entire function whenever $f \in \operatorname{LP}(\mathbb{C} ; \mathbb{R})$ and $\beta\left(F_{T}\right) \beta(f)<\frac{1}{4}$, where the various betas are the order two characteristics of the indicated functions [9] (see also [3, p. 360] or [5, p. 147]); in this case, it is easy to see that either $T[f] \in \operatorname{LP}(\mathbb{C} ; \mathbb{R})$ or $T[f]=0$. The constant $\frac{1}{4}$ appearing here is sharp.

A consequence of the Pólya-Benz theorem for multiplicative differentiation. The fact that the Pólya-Benz theorem extends beyond the polynomials means that we may apply it to the class of entire functions of finite exponential type with only real zeros which have a fixed real period. Such functions have a particularly simple structure: after applying the exponential mapping to the plane after an appropriate rotation, we obtain the class $\mathcal{R}_{s}\left(\mathbb{C}_{*} ; \mathbb{T}\right)$, which consists of rational functions with (1) poles (possibly multiple) only at the origin and at infinity, (2) zeros only on the unit circle $\mathbb{T}$, and (3) real values along $\mathbb{T}$. Condition (3) means that the functions $f$ in $\mathcal{R}_{s}\left(\mathbb{C}_{*} ; \mathbb{T}\right)$ have the following symmetry property with respect to reflection in the unit circle:

$$
\bar{f}\left(\frac{1}{\bar{z}}\right)=f(z), \quad z \in \mathbb{C}_{*} .
$$


Here $\mathbb{C}_{*}$ is the multiplicative plane $\mathbb{C} \backslash\{0\}$. We write $\operatorname{LP}(\mathbb{C} ; i \mathbb{R})$ for the collection of all entire functions $F(z)$ for which $F(i z)$ belongs to $\operatorname{LP}(\mathbb{C} ; \mathbb{R})$.

Corollary 1.3. Let $F$ be an entire function in the class $\operatorname{LP}(\mathbb{C} ; i \mathbb{R})$. Let $f$ be a rational function in the class $\mathcal{R}_{s}\left(\mathbb{C}_{*} ; \mathbb{T}\right)$. Then the function $F\left(D_{*}\right) f$ belongs to $\mathcal{R}_{s}\left(\mathbb{C}_{*} ; \mathbb{T}\right) \cup\{0\}$.

The result is a fairly immediate consequence of the Pólya-Benz theorem; we leave it to the interested reader to reconstruct the proof.

It would be of interest to investigate the extent to which an operator $T$ which commutes with $D_{*}$ and preserves $\mathcal{R}_{s}\left(\mathbb{C}_{*} ; \mathbb{T}\right) \cup\{0\}$ must be of the form $F\left(D_{*}\right)$ with $F \in \mathrm{LP}(\mathbb{C} ; i \mathbb{R})$. The usual proof of the necessity which works for the Pólya-Benz theorem seems to fail here.

A Pólya-Schur type theorem for inverted plane differentiation. We now turn our attention to operators $T: \mathcal{P}(\mathbb{C}) \rightarrow \mathcal{P}(\mathbb{C})$ that commute with $D_{\sharp}$, the inverted plane differentiation operator. One shows that such an operator $T$ is of the form

$$
T=F\left(D_{\sharp}\right)+L,
$$

where $F$ is a complex polynomial and $L$ is a rank one operator of the form

$$
L[p](z)=\lambda_{0} \widehat{p}(0)+\lambda_{1} \widehat{p}(1), \quad p(z)=\sum_{n=0}^{+\infty} \widehat{p}(n) z^{n},
$$

where $\lambda_{0}$ and $\lambda_{1}$ are complex constants.

We turn to our main result, the characterization of the real zero preserving operators that commute with $D_{\sharp}$.

Theorem 1.4. Let $T: \mathcal{P}(\mathbb{C}) \rightarrow \mathcal{P}(\mathbb{C})$ be a linear operator which commutes with $D_{\sharp}$. Then $T$ preserves real zeros if and only if it is of one of the following forms:

(a) $T[p](z)=\lambda_{0} \widehat{p}(0)+\lambda_{1} \widehat{p}(1)$, where $\lambda_{0}, \lambda_{1}$ are complex constants, or

(b) $T=\alpha D_{\#}^{N}$ for some complex constant $\alpha$ and some integer $N=0,1,2, \ldots$.

This result is in striking contrast with Theorems 1.1 and 1.2, where there was a rich collection of real zero preserving operators that commute with $D_{*}$ or with $D$. The "test points" that we should test $T$ upon to get Theorem 1.4 are the first, second, and third degree polynomials with real zeros. We suggest the following terminology: a real zero preserving operator $T$ on the polynomials is real zero meager if the only operators that (1) commute with it and (2) preserve real zeros 
are of the form $\alpha T^{n}$, for some complex scalar $\alpha$ and a nonnegative integer $n$. In particular, we see that $D_{\sharp}$ is almost real zero meager. One would tend to suspect that most real zero preserving operators are in fact real zero meager.

We should point out the following interpretation of Theorem 1.4. Let $\mathbb{C}_{\infty}=$ $\mathbb{C} \cup\{\infty\}$ denote the extended complex plane (the Riemann sphere), and for a given point $\lambda \in \mathbb{C}_{\infty}$, let $\mathcal{R}\left(\mathbb{C}_{\infty} \backslash\{\lambda\} ; \mathbb{R}\right)$ denote the collection of rational functions with a (possibly multiple) pole at the point $\lambda$ only, whose zeros are all located along the real line. Then, if $\lambda \in \mathbb{C} \backslash \mathbb{R}$, the differentiation operator $D$ does not preserve the class $\mathcal{R}\left(\mathbb{C}_{\infty} \backslash\{\lambda\} ; \mathbb{R}\right) \cup\{0\}$. Moreover, if $\lambda \in \mathbb{R}$, then $D$ preserves $\mathcal{R}\left(\mathbb{C}_{\infty} \backslash\{\lambda\} ; \mathbb{R}\right) \cup\{0\}$, but essentially all operators that commute with $D$ and preserve $\mathcal{R}\left(\mathbb{C}_{\infty} \backslash\{\lambda\} ; \mathbb{R}\right) \cup\{0\}$ are constant multiples of powers of $D$. This follows from Theorem 1.4, since after a translation (which commutes with $D$ ) we may reduce to the case $\lambda=0$, which is equivalent to studying $D_{\sharp}$ on $\operatorname{LP}(\mathbb{C} ; \mathbb{R}) \cup\{0\}$. Finally, the remaining case $\lambda=\infty$ is treated by the Pólya-Benz theorem, which contrasts with the other values of $\lambda$ in having a rich collection of real zero preserving operators that commute with $D$.

\section{The inverted plane}

In this section, we consider the weighted forward shift operator $D_{\#}$ defined on $\mathcal{P}(\mathbb{C})$ by

$$
D_{\#} p(z)=z^{2} D p(z)=z^{2} p^{\prime}(z) .
$$

We show that, as for the preservation of real zeros, the operators in the commutant of $D_{\#}$ behave somewhat differently from those of the commutants of the additive or multiplicative differentiation operators ( $D$ and $D_{*}$ ).

We turn to the description of the commutant of $D_{\#}$.

Proposition 2.1. Let $T: \mathcal{P}(\mathbb{C}) \rightarrow \mathcal{P}(\mathbb{C})$ be a linear operator which commutes with $D_{\#}$. Then there exists a polynomial $F$ and two constants $\lambda_{0}, \lambda_{1} \in \mathbb{C}$ which are determined uniquely by $T$ such that

$$
T[p](z)=F\left(D_{\#}\right)[p](z)+\lambda_{0} \widehat{p}(0)+\lambda_{1} \widehat{p}(1),
$$

where $p(z)=\sum_{n=0}^{+\infty} \widehat{p}(n) z^{n}$. Moreover, if we introduce the polynomial $q=T[z]$, then $F$ is given by

$$
F(z)=\sum_{n=0}^{+\infty} \frac{\widehat{q}(n+1)}{n !} z^{n} .
$$

Finally, any operator $T$ of the above form commutes with $D_{\sharp}$.

Proof. We begin with the following simple observation, based on the fact that

$$
D_{\#}^{n} z=n ! z^{n+1}, \quad n=0,1,2, \ldots
$$


If $p$ is any polynomial and $r \in \mathcal{P}(\mathbb{C})$ is defined by $r(z)=p\left(D_{\#}\right) z$, then

$$
r(z)=p\left(D_{\#}\right)[z]=\sum_{n=0}^{+\infty} \widehat{p}(n) D_{\#}^{n}[z]=\sum_{n=0}^{+\infty} \widehat{p}(n) n ! z^{n+1}
$$

consequently, the coefficients of $p$ and $r$ are related via

$$
\widehat{r}(n+1)=n ! \widehat{p}(n), \quad n=0,1,2, \ldots, \quad \widehat{r}(0)=0 .
$$

Now let $T$ be an operator that commutes with $D_{\# \text {. As }}$

$$
D_{\#} T[1]=T D_{\#}[1]=0,
$$

it follows that $T[1]=c_{0}$, with $c_{0}$ a complex constant. Set $q=T[z]$, where

$$
q(z)=\sum_{n=0}^{+\infty} \widehat{q}(n) z^{n}
$$

and define

$$
F(z)=\sum_{n=0}^{+\infty} \widehat{F}(n) z^{n}
$$

where the coefficients $\widehat{F}(n)$ are given by

$$
\widehat{F}(n)=\frac{1}{n !} \widehat{q}(n+1), \quad n=0,1,2, \ldots
$$

Then, by the observation made at the beginning of the proof, we have that

$$
F\left(D_{\#}\right)[z]=q(z)-\widehat{q}(0) .
$$

Hence, the operator $L=T-F\left(D_{\#}\right)$ satisfies

$$
L[1]=c_{0}-\widehat{F}(0), \quad L[z]=q(z)-(q(z)-\widehat{q}(0))=\widehat{q}(0),
$$

and for $n=2,3,4, \ldots$,

$$
\begin{aligned}
L\left[z^{n}\right]=\frac{1}{(n-1) !}\left(T-F\left(D_{\#}\right)\right) D_{\#}^{n-1}[z] & =\frac{1}{(n-1) !} D_{\#}^{n-1} L[z] \\
& =\frac{1}{(n-1) !} D_{\#}^{n-1}[\widehat{q}(0)]=0 .
\end{aligned}
$$

In other words, for any polynomial $p, L p(z)=\lambda_{0} \widehat{p}(0)+\lambda_{1} \widehat{p}(1)$, where $\lambda_{0}=c_{0}-\widehat{F}(0)$ and $\lambda_{1}=\widehat{q}(0)$.

Finally, it is a simple exercise to verify that an operator $T$ of the given form

$$
T[p](z)=F\left(D_{\#}\right)[p](z)+\lambda_{0} \widehat{p}(0)+\lambda_{1} \widehat{p}(1)
$$

commutes with $D_{\sharp}$. 
Remark 2.2. (a) We first note that the above proposition remains valid with essentially the same proof if we replace the operator $D_{\#}$ by any other weighted forward shift, so long as we make the necessary modifications in the definition of $F$. Here, by a weighted forward shift we mean an operator $S: \mathcal{P}(\mathbb{C}) \rightarrow \mathcal{P}(\mathbb{C})$ which satisfies

$$
S[1]=0, \quad S\left[z^{n}\right]=\alpha_{n} z^{n+1}, \quad n=1,2,3, \ldots,
$$

for a positive weight sequence $\alpha_{n}, n=1,2,3 \ldots$.

(b) Given an operator $T$ that commutes with $D_{\#}$, there is an alternative way to find the polynomial $F$ appearing in our proposition. Indeed, since for each $N=2,3,4, \ldots$ we have $T z^{N}=F\left(D_{\#}\right) z^{N}$, it follows that

$$
R(z)=z^{-N} T\left[z^{N}\right]=\sum_{n=0}^{+\infty} \widehat{F}(n) \frac{(N+n-1) !}{(N-1) !} z^{n},
$$

and hence

$$
R\left(\frac{z}{N}\right)=\sum_{n=0}^{+\infty} \widehat{F}(n) \frac{(N+n-1) !}{N^{n}(N-1) !} z^{n} .
$$

Now, letting $N \rightarrow+\infty$, we obtain

$$
\lim _{N \rightarrow \infty} R\left(\frac{z}{N}\right)=\sum_{n=0}^{+\infty} \widehat{F}(n) z^{n}=F(z) .
$$

In particular, it follows that if $T$ preserves real zeros, then the polynomial $F$ must have only real zeros. Again, this reasoning remains valid for many other weighted forward shifts. In fact, the above argument - on how to obtain the function $F(z)$ - can be applied to the commutant of any weighted forward shift operator $S: \mathcal{P}(\mathbb{C}) \rightarrow \mathcal{P}(\mathbb{C})$ of the type mentioned in part (a), provided the associated positive numbers $\alpha_{n}$ have the following property: there exists a sequence of real parameters $\left\{\beta_{N}\right\}_{N}$ such that for each $n=1,2,3, \ldots$,

$$
\sum_{k=0}^{n-1} \log \alpha_{N+k}=n \beta_{N}+o(1) \quad \text { as } N \rightarrow+\infty .
$$

For the convenience of the reader, we restate the main result.

Theorem 1.4. Let $T: \mathcal{P}(\mathbb{C}) \rightarrow \mathcal{P}(\mathbb{C})$ be a linear operator which commutes with $D_{\#}$. Then $T$ preserves real zeros if and only if $T$ is of one of the following forms: 
(a) $T[p](z)=\lambda_{0} \widehat{p}(0)+\lambda_{1} \widehat{p}(1)$, where $p(z)=\sum_{n=0}^{+\infty} \widehat{p}(n) z^{n}$, and $\lambda_{0}, \lambda_{1}$ are complex constants, or

(b) $T=\alpha D_{\#}^{N}$ for some complex constant $\alpha$ and nonnegative integer $N$.

Proof. We first consider the sufficiency of the conditions (a) and (b). If $T$ is of the form stipulated by (a), then $T$ maps all polynomials to constants and therefore necessarily preserves real zeros. By the Gauss-Lucas theorem, $D_{\#}$ preserves real zeros; and hence every operator $T$ of the form stipulated by (b) preserves real zeros as well.

We turn to the necessity part. We suppose that $T$ preserves real zeros and that it is not the zero operator. We use Proposition 2.1 to write $T$ in the form

$$
T[p](z)=F\left(D_{\#}\right)[p](z)+\lambda_{0} \widehat{p}(0)+\lambda_{1} \widehat{p}(\mathbf{1}) .
$$

If $F$ is the zero polynomial, we are in situation (a). It remains to consider the case that $F$ is not the zero polynomial. We first consider constant $F$, that is, $F(z)=\widehat{F}(0) \neq 0$. By replacing $T$ with a suitable constant multiple of itself, we may assume that $\widehat{F}(0)=1$. We apply $T$ to the test functions $p_{\alpha}(z)=(z-\alpha)^{3}$, where $\alpha$ is a real parameter, and obtain

$$
T\left[p_{\alpha}\right](z)=(z-\alpha)^{3}-\lambda_{0} \alpha^{3}+3 \lambda_{1} \alpha^{2} .
$$

This function has real zeros for all real $\alpha$ if and only if

$$
\alpha^{2}\left(\lambda_{0} \alpha-3 \lambda_{1}\right)=0
$$

for all $\alpha \in \mathbb{R}$. Clearly, this implies that $\lambda_{0}=\lambda_{1}=0$, making $T$ a multiple of the identity operator. We turn to nonconstant $F$. Let us first consider the test polynomials $q_{\alpha}(z)=z-\alpha$, with real $\alpha$, and note that

$$
T\left[q_{\alpha}\right](z)=T[z]-\alpha T[1]=\sum_{n=0}^{+\infty} \widehat{F}(n) n ! z^{n+1}+\lambda_{1}-\alpha\left(\widehat{F}(0)+\lambda_{0}\right) .
$$

We claim that $T\left[q_{\alpha}\right]$ cannot have real zeros for all $\alpha \in \mathbb{R}$ unless

$$
T[1]=\widehat{F}(0)+\lambda_{0}=0 .
$$

Indeed, if $d(1 \leq d<+\infty)$ is the degree of the polynomial $F$, then

$$
\frac{1}{\alpha} T\left[q_{\alpha}\right]\left(|\alpha|^{1 /(d+1)} z\right) \rightarrow \widehat{F}(d) z^{d+1}-\left(\widehat{F}(0)+\lambda_{0}\right) \quad \text { as } \alpha \rightarrow+\infty,
$$

uniformly on compact subsets of $\mathbb{C}$. This justifies our claim for $2 \leq d<+\infty$, as the right-hand side develops complex roots unless $(2.2)$ holds. To treat the 
remaining case $d=1$, we consider the above limit as $\alpha \rightarrow-\infty$ as well, and reach the same conclusion (2.2). Thus, we may restrict our attention to operators $T$ with (2.2). Note that the function $T[z]$ must have only real zeros, for if it were to vanish identically, we would deduce that $F(z) \equiv 0$. We next consider the test polynomials

$$
p_{\alpha, \beta}(z)=(z-\alpha)(z-\beta)=z^{2}-(\alpha+\beta) z+\alpha \beta,
$$

where $\alpha, \beta$ are real. Then

$$
T\left[p_{\alpha, \beta}\right](z)=T\left[z^{2}\right]-(\alpha+\beta) T[z]+\alpha \beta T[1]=T\left[z^{2}\right]-(\alpha+\beta) T[z]
$$

should have only real zeros for all values of $\alpha, \beta \in \mathbb{R}$. Expressed differently, the rational function

$$
Q(z)=\frac{T\left[z^{2}\right]}{T[z]}
$$

has the property that the preimage of the real axis is contained in the real axis. At this point, the following special property of the operator $D_{\#}$ plays an essential role. Since

$$
T\left[z^{2}\right]=\sum_{n=0}^{+\infty} \widehat{F}(n)(n+1) ! z^{n+2}, \quad T[z]=\sum_{n=0}^{+\infty} \widehat{F}(n) n ! z^{n+1}+\lambda_{1},
$$

we have $T\left[z^{2}\right]=z^{2} D T[z]=z^{2} T[z]^{\prime}$. This implies that if $a_{1} \leq a_{2} \leq \cdots \leq a_{d}$ are the (real) roots of the polynomial $T[z]$, then

$$
Q(z)=\frac{T\left[z^{2}\right]}{T[z]}=z^{2} \frac{T[z]^{\prime}}{T[z]}=z^{2} \sum_{k=1}^{d} \frac{1}{z-a_{k}} .
$$

We claim that the fact that $Q^{-1}(\mathbb{R}) \subset \mathbb{R}$ implies that

$$
a_{1}=a_{2}=\cdots=a_{d}=0 .
$$

To see this, assume first that $a_{d} \neq 0$. Note that in view of the form of $Q$, this function $Q$ maps the real line - except for the points $a_{1}, a_{2}, \ldots, a_{d}$ - into the real line. We quickly find that

$$
Q(x) \rightarrow+\infty \quad \text { as } x \rightarrow a_{d}^{+}, \quad Q(x) \rightarrow+\infty \quad \text { as } x \rightarrow+\infty .
$$

This means that on the interval $] a_{d},+\infty\left[, Q(x)\right.$ must assume a minimum value $q_{0}$ at some point $\left.x=b_{0} \in\right] a_{d},+\infty\left[\right.$. For $q$ with $q_{0}<q<+\infty$, there are then at least two real roots of the equation $Q(z)=q$ in the interval $] a_{d},+\infty[$, at least one on each side of $b_{0}$. However, for $q$ with $-\infty<q<q_{0}$, there are-no real roots of the equation $Q(z)=q$ that fall in the interval $] a_{d},+\infty[$. It is well-known that the collection of 
complex roots of the equation $Q(z)=q$ moves continuously with the parameter $q$, which allows us to conclude that there must be complex non-real roots of $Q(z)=q$ for $q<q_{0}$ close to $q_{0}$. This contradicts the assumption that $Q^{-1}(\mathbb{R}) \subset \mathbb{R}$. Hence, we are left only with the possibility that $a_{d}=0$. By a similar argument, we also have $a_{1}=0$, so that all the roots of $T[z]$ are at the origin, and $T[z]=\beta z^{d}$ for some nonzero complex constant $\beta$. This implies that $F(z)=\alpha z^{N}$ for some nonzero complex constant $\alpha$ with $N=d$; moreover, we also see that $\lambda_{1}=0$. The result follows.

\title{
Acknowledgements
}

Håkan Hedenmalm thanks the Göran Gustafsson Foundation for the financial support that made the present paper possible. We also thank the referee for several valuable comments.

\section{REFERENCES}

[1] E. W. Benz, Ubber lineare verschiebungstreue Funktionaloperatoren und die Nullstellen ganzer Funktionen, Comment. Math. Helv. 7 (1934), 243-289.

[2] R. P. Boas, Jr., Entire Functions, Academic Press, New York, 1954.

[3] B. Ja. Levin, Distribution of Zeros of Entire Functions, Revised edition. Translations of Mathematical Monographs, 5, American Mathematical Society, Providence, R.I., 1980.

[4] G. Pólya, Über Annäherung durch Polynome mit lauter reellen Wurzeln, Rend. Circ. Mat. Palermo 36 (1913), 279-295.

[5] R. P. Boas, ed. George Pólya: Collected Papers. Vol. II: Location of Zeros, MIT Press, Cambridge, Mass.-London, 1974.

[6] G. Pólya and J. Schur, Über zwei Arten von Faktorenfolgen in der Theorie der algebraischen Gleichungen, J. Reine Angew. Math. 144 (1914), 89-113.

[7] J. Schur, Zwei Sätze über algebraische Gleichungen mit lauter reellen Wurzeln, J. Reine Angew. Math. 144 (1914), 75-88.

[8] Issai Schur: Gesammelte Abhandlungen. Band II (German), Herausgegeben von Alfred Brauer und Hans Rohrbach, Springer-Verlag, Berlin-New York, 1973.

[9] P. C. Sikkema, Differential Operators and Differential Equations of Infinite Order with Constant Coefficients. Researches in Connection with Integral Functions of Finite Order, P. Noordhoff N. V., Gröningen-Djakarta, 1953.

\author{
A. Aleman \\ DEPARTMENT OF MATHEMATICS \\ LUND UNIVERSITY, BOX 118 \\ S-22100 LUND, SWEDEN \\ email: alemanQmaths.Ith.se
}


D. Beliaev and H. Hedenmalm DEPARTMENT OF MATHEMATICS

THE ROYAL INSTITUTE, OF TECHNOLOGY

S-10044 STOCKHOLM, SWEDEN email: beliaev@math.kth.se, haakanh@math.kth.se

(Received March 2, 2003 and in revised form May 25, 2003) 